\title{
Clinical Characteristics and Factors Associated with Disease Progression in Chinese Patients with Connective Tissue Disease and Pulmonary Arterial Hypertension
}

\author{
Klinische Merkmale und Einflussfaktoren auf den \\ Krankheitsverlauf bei chinesischen Patienten mit \\ Bindegewebserkrankungen und pulmonal-arterieller Hypertonie
}

Authors

Haichao Zhang, Xiaoyan Liu xiaoyan

\section{Affiliation}

Pharmacy, Shanghai Jiao Tong University School of Medicine Affiliated Renji Hospital, Shanghai, China

Keywords

pulmonary arterial hypertension, systemic lupus erythematosus, connective tissue disease

\section{Schlüsselwörter \\ Lupus erythematodes, Bindegewebserkrankung, pulmonal- arterielle Hypertonie}

Online publiziert: 7.4 .2020

Bibliography

Akt Rheumatol 2020; 45: 475-479

DOI 10.1055/a-1135-8548

ISSN 0341-051X

(c) 2020. Thieme. All rights reserved.

Georg Thieme Verlag KG, Rüdigerstraße 14,

70469 Stuttgart, Germany

Correspondence

Dr. Xiaoyan Liu xiaoyan

pharmacy

Shanghai Jiao Tong University School of Medicine

Affiliated Renji Hospital

No.1630 Dongfang Road

200127 Shanghai

China

Tel : + 8617317509930, Fax : 021-58752345

liuxiaoyanrj@163.com

\section{ABSTRACT}

Objective To clarify the characteristics, survival, and predictors of mortality in Chinese patients with pulmonary arterial hypertension (PAH) associated with connective tissue disease (CTD).
Methods This single-cohort study involved 67 CTD-PAH patients who visited Shanghai Renji Hospital between January 2011 and January 2019. Baseline clinical features and hemodynamic parameters were recorded. Logistic regression was used to determine the independent factors associated with mPAP improvement.

Results SLE was the major underlying CTD, affecting $70 \%$ of PAH patients, whereas systemic sclerosis was less common (6\%). Modern PAH drug use was associated with a favorable outcome and mPAP improvement.

Conclusion The majority of the CTD-PAH population in China suffers from SLE in contrast to the CTD-PAH patients in the USA and Europe. Pulmonary vasodilation treatment has improved the mPAP in these patients.

\section{ZUSAMMENFASSUNG}

Zielsetzung Untersuchung der klinischen Merkmale, des Überlebens und der Prädiktoren der Mortalität bei chinesischen Patienten mit pulmonal-arterieller Hypertonie (PAH) in Verbindung mit einer Bindegewebserkrankung (CTD).

Methoden In dieser Einzelkohortenstudie wurden 67 CTDPAH-Patienten untersucht, die im Zeitraum von Januar 2011 bis Januar 2019 im Shanghai Renji Hospital behandelt wurden. Dabei wurden klinische Ausgangsmerkmale und hämodynamische Parameter erfasst. Mittels logistischer Regression wurden die mit einer Verbesserung des mPAP einhergehenden unabhängigen Faktoren ermittelt.

Ergebnis SLE war die Hauptursache für CTD und betraf $70 \%$ der PAH-Patienten, wohingegen die systemische Sklerose seltener auftrat (6\%). Die Anwendung einer modernen $\mathrm{PAH}$ Medikation führte zu günstigen Ergebnissen und einer Verbesserung des mPAP.

Schlussfolgerung Im Gegensatz zu den CTD-PAH-Patienten in den USA und Europa leidet die Mehrheit der CTD-PAH-Population in China an SLE. Die pulmonale Vasodilatation führte bei diesen Patienten zu einer Verbesserung des mPAP. 


\section{Introduction}

Pulmonary arterial hypertension (PAH) is an intractable condition that, in patients with connective tissue disease (CTD), has progressive debilitating symptoms and a poor prognosis [1]. Compared with those with idiopathic PAH (IPAH), patients with PAH associated with CTD (CTD-PAH) [2,3] have poorer outcomes and worse survival. In cohort studies conducted in the USA and Europe, the majority of patients with CTD-PAH had systemic sclerosis (SSC) $[2,4,5]$. However, in our clinical experience, systemic lupus erythematosus (SLE) is the most common CTD in China.

Over the past decade, $\mathrm{PAH}$-specific vasodilation agents, including prostanoids, endothelin receptor antagonists and phosphodiesterase 5 inhibitors, have become available for clinical use. Consequently, several studies that primarily enrolled SSc patients reported improvement in short-term survival $[2,4,6]$. However, few data are available on the improvement in survival of Chinese patients with CTD-PAH in the modern treatment era.

In this study, we evaluated the clinical characteristics and prognosis of patients with CTD-PAH who were diagnosed and monitored at our hospital in China. We also investigated the impact of $\mathrm{PAH}$ drugs on survival and the improvement of mean pulmonary arterial pressure (mPAP).

\section{Materials and Methods}

\section{Patient cohort}

We included all patients with CTD-PAH diagnosed at our hospital from January 2011 to January 2019. All included patients had CTD diagnosis, and PAH was diagnosed based on the following criteria: (1) a mPAP over $25 \mathrm{mmHg}$ at rest by right heart catheterization; (2) exclusion of left-sided heart disease; (3) exclusion of chronic thromboembolism; and (4) exclusion of advanced interstitial lung disease (ILD), determined by forced vital capacity predicted < 70 \% [7]. Patient demographics, clinical variables, and cardiac and pulmonary assessments were obtained.

\section{Outcome}

The primary outcome of the study was the time from PAH diagnosis to death from all causes. Patients alive as of January 1, 2019, were censored. The date of PAH diagnosis was defined as the date of the right heart catheterization, and the date of CTD diagnosis was defined as the date of a diagnosis of CTD made by a physician. Dates of death were obtained from the electronic medical record. Secondary outcomes included sex differences in age at PAH diagnosis, time from CTD diagnosis to PAH diagnosis, the subtype of CTD and mPAP changes.

\section{Analysis}

Patient characteristics at baseline were reported as the mean \pm standard deviation (SD) for continuous variables and proportions for categorical variables. Differences in baseline mPAP between different subtypes of CTD were compared using Student's t-test. Differences between the 4 subtypes of CTD-PAH were compared using analysis of variance. To identify variables that predict improvements of mPAP in patients with CTD-PAH, a multivariate analysis was performed. A two-tailed P-value $\leq 0.05$ was considered statistically sig- nificant. All statistical analyses were performed using Rstudio 1.2.1335.

\section{Results}

A total of 67 patients with CTD-PAH were included. Patient characteristics at baseline are summarized in $>$ Table 1. Our CTD-PAH cohort mainly consisted of women (94\%) with 4 disease subtypes (SLE, mixed connective tissue disease (MCTD), Sjogren's syndrome (SS), and SSc). The mean age of the patients at PAH diagnosis was $36.66 \pm$ 10.39, while the mean age at CTD diagnosis was $30.00 \pm 11.20$. The mean mPAP at PAH diagnosis was $47.3 \pm 10.5$. After PAH diagnosis, 5 patients were not prescribed any pulmonary vasodilation therapy, 27 patients were prescribed monotherapy and 11 patients were prescribed combination therapy.

Because only one person died during the follow-up, we decided to not use the KM curves. As a result, we recorded the mPAP three years after the PAH diagnosis and calculated the differences between the mPAP at diagnosis and the mPAP three years after diagnosis. Then, we performed an analysis of variance to determine whether a combination vasodilation therapy should be used. Be-

Table 1 Patients characteristics at baseline.

\begin{tabular}{|l|l|}
\hline Characteristics & Mean+ SD or n(\%) \\
\hline Total number of patients & 67 \\
\hline Female & $63(94 \%)$ \\
\hline Age at PAH diagnosis, years & $36.66 \pm 10.39$ \\
\hline Age at CTD diagnosis, years & $30.00 \pm 11.20$ \\
\hline Underlying CTD & \\
\hline SLE & $47(70 \%)$ \\
\hline MCTD & $9(14 \%)$ \\
\hline Sjogren's syndrome & $7(10 \%)$ \\
\hline SSC & $4(6 \%)$ \\
\hline ANA(+) & $67(100 \%)$ \\
\hline Anti-U1RNP(+) & $36(54 \%)$ \\
\hline Anti-SSA (+) & $48(72 \%)$ \\
\hline Anti-SSB(+) & $5(7 \%)$ \\
\hline Anti-dsDNA(+) & $14(21 \%)$ \\
\hline mPAP at PAH diagnosis & $47.3 \pm 10.5$ \\
\hline PVR, Wood units & $13.1 \pm 6.3$ \\
\hline BNP, pg/ml & $235.8 \pm 379.6$ \\
\hline Pulmonary vasodilator therapy & \\
\hline Unknown & $24(37 \%)$ \\
\hline Untreated & $5(7 \%)$ \\
\hline Monotherapy & $27(40 \%)$ \\
\hline Combination therapy & $11(16 \%)$ \\
\hline
\end{tabular}

CTD: connective tissue disease, PAH: pulmonary arterial hypertension. SLE: systemic lupus erythematosus, MCTD: mixed connective tissue disease, SSc: systemic sclerosis, mPAP: mean pulmonary arterial pressure, PVR: pulmonary vascular resistance, BNP: brain natriuretic peptide 
cause of missing data, 43 patients were included in this analysis. As monotherapy, sildenafil (15) was the most commonly prescribed drug, followed by bosentan (10). Other prescribed monotherapies included tadalafil (1) and vardenafil (1). The most common combination was bosentan and sildenafil (6). All patients were prescribed CTD therapies. Compared with the untreated group, both the monotherapy group $(P=0.001)$ and the combination therapy group $(P=0.055)$ had a better prognosis. However, the results did not show any improvement between the combination therapy group and the monotherapy group $(P=0.312)$.

Baseline demographic, clinical and hemodynamic parameters were compared according to the underlying CTD; the results are shown in > Table 2. Among the 4 CTD groups, patients with SLE were the youngest at PAH diagnosis, followed by patients with MCTD and SS, while the patients in the SSc group were the oldest. Renal disorder was exclusively found in patients with SLE at the diagnosis of $\mathrm{PAH}$. There were no statistically significant differences in the mPAP among the 4 groups.

To identify variables that predict improvements of mPAP in patients with CTD-PAH, we performed a multivariate analysis to find prognostic factors from the baseline characteristics and treatment regimens. We first recognized all of the missing data, as shown in - Fig. 1. Then, we used a multiple imputation (MI) method to complete the data $(m=30)$. MI provides an approach to missing values that's based on repeated simulations. In MI, a set of of complete datasets (typically 3 to 10 while we used 30 datasets, indicated by $\mathrm{m}=30$ ) is generated from an existing dataset containing missing values. Monte Carlo methods are used to fill in the missing data in each of the simulated datasets. Standard statistical methods are applied to each of the simulated datasets, and the outcomes are combined to provide estimated results and confidence intervals that take into account the uncertainty introduced by the missing values [8]. After that, we performed the logistic regression analysis, and the results are shown in $>$ Table 3 . As shown in the table, only the use of any PAH drug was a factor for favorable improvement of the mPAP.

\section{Discussion}

$\mathrm{PAH}$ is an incurable comorbidity in patients with CTD. CTD-PAH presents similar pulmonary and cardiac manifestations to other forms of $\mathrm{PAH}$ but has a worse prognosis compared with idiopathic $\mathrm{PAH}$ [9]. This study, conducted in our hospital, is a cohort study of Chinese patients with CTD-PAH. We found that the characteristics of our patients with CTD-PAH included high frequencies of SLE as the underlying CTD, which is in sharp contrast to cohort studies conducted in the USA and Europe where SSc patients were found to comprise $>70 \%$ of the patients with CTD-PAH $[2,4-5]$.

The mean age at PAH diagnosis in China was 36.7, which was much younger than the 49-56 year range reported for the US and European cohorts $[2,4,5]$. This difference might be explained by a higher frequency of SLE among our patients with CTD-PAH. Only a minor group of our patients with CTD-PAH had SSc, whose mean age was 54.8, which is consistent with features reported in the USA and European patients with SSc-PAH. The distribution of underlying CTDs in our study was almost concordant with the studies performed in Japan and China $[10,11]$. Additionally, PAH was reported as the third leading cause of death in Korean patients with SLE [12]. The reasons for these ethnic differences in the distribution of underlying CTDs in patients with CTD-PAH remain unclear. However, genetic and environmental factors may play a role.

$\mathrm{PAH}$ is one of the major causes of mortality in patients with CTD. In China, most patients will not be diagnosed with $\mathrm{PAH}$ until right ventricular dysfunction has developed. In our study, the mean mPAP at PAH diagnosis was $47.3 \mathrm{mmHg}$, which is a considerably high level. It has been proposed that early detection and therapeutic intervention are critical for improving the survival rates of patients with PAH [13]. Any deterioration in pulmonary hemodynamics recognized early could help to initiate pulmonary vasodilation therapy. Because the smallest mean time between the onset of CTD and $\mathrm{PAH}$ diagnosis was 3.3 years, as shown in this study, routine echocardiography screening for PAH three years after a CTD diagnosis is recommended for all patients regardless of whether symptoms are detectable. If the result of echocardiography screening

- Table 2 Demographic and clinical characteristics in patients with CTD-PAH

\begin{tabular}{|c|c|c|c|c|c|}
\hline Demographic and clinical features & SLE $(n=47)$ & MCTD (n=9) & SS $(n=7)$ & $\operatorname{SSc}(n=4)$ & Overall $\mathbf{P}$ \\
\hline Female (\%) & 94 & 100 & 86 & 100 & 0.65 \\
\hline Age at PAH diagnosis (years) & $34.7 \pm 9.1$ & $37.2 \pm 7.8$ & $38.8 \pm 11.4$ & $54.8 \pm 13.4$ & $<0.01$ \\
\hline $\begin{array}{l}\text { Time between CTD onset and PAH } \\
\text { diagnosis (years) }\end{array}$ & $7.4 \pm 7.0$ & $3.3 \pm 3.4$ & $4.5 \pm 5.3$ & $5.0 \pm 5.2$ & 0.27 \\
\hline Renal disorder (\%) & 26 & 0 & 0 & 0 & 0.02 \\
\hline $\operatorname{ANA}(+)(\%)$ & 100 & 100 & 100 & 100 & \\
\hline Anti-U1RNP(+)(\%) & 55 & 100 & 14 & 0 & 0.01 \\
\hline Aiti-SSA(+)(\%) & 79 & 67 & 28 & 75 & 0.05 \\
\hline Anti-SSB $(+)(\%)$ & 8 & 11 & 0 & 0 & 0.77 \\
\hline Anti-dsDNA(+)(\%) & 23 & 22 & 0 & 25 & 0.56 \\
\hline mPAP & $46.5 \pm 10.2$ & $45.4 \pm 8.3$ & $52.4 \pm 9.5$ & $52.1 \pm 22.1$ & 0.70 \\
\hline
\end{tabular}

CTD: connective tissue disease, PAH: pulmonary arterial hypertension. SLE: systemic lupus erythematosus, MCTD: mixed connective tissue disease, SS: Sjogren's syndrome, SSc: systemic sclerosis, mPAP: mean pulmonary arterial pressure, 


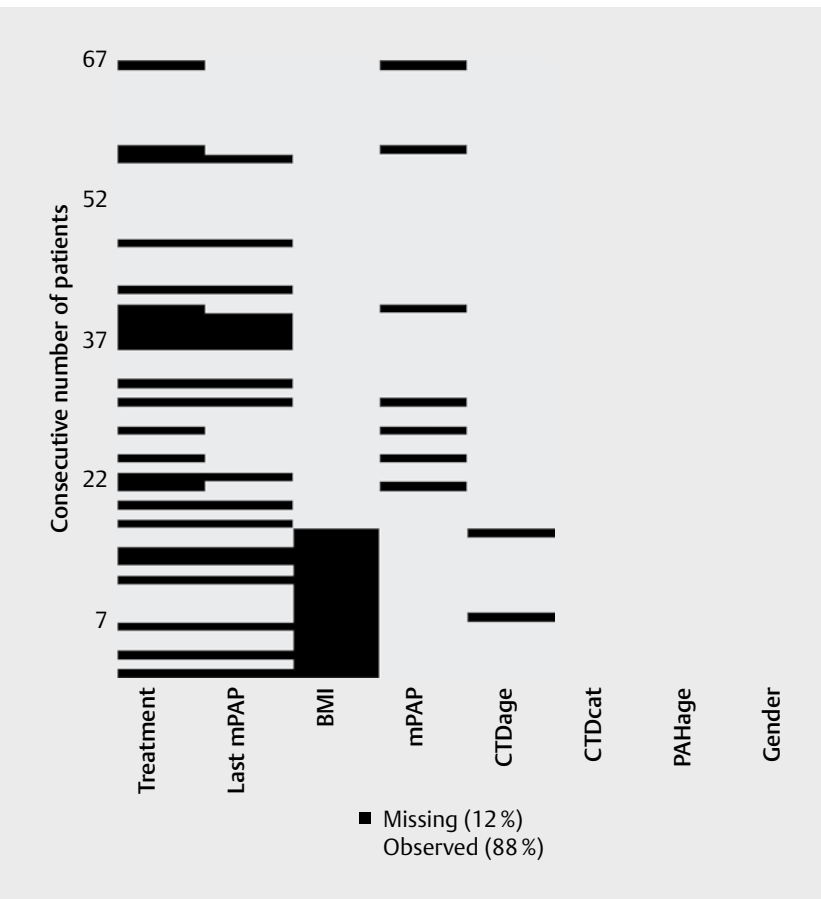

× Fig. 1 Missing values vs. observed.

- Table 3 Independent predictors of mPAP improvement, obtained by multivariate analysis.

\begin{tabular}{|c|c|c|c|}
\hline & Estimate & Statistic & p.value \\
\hline (Intercept) & 70.48 & 1.80 & 0.08 \\
\hline Gender1 & 10.25 & 0.43 & 0.66 \\
\hline PAHage & 0.08 & 0.10 & 0.91 \\
\hline CTDage & -0.77 & -1.01 & 0.31 \\
\hline BMI & -0.49 & -0.28 & 0.77 \\
\hline MCTD group * & 1.10 & 0.07 & 0.93 \\
\hline SS group * & 1.03 & 0.07 & 0.94 \\
\hline SSc group * & -0.35 & -0.01 & 0.98 \\
\hline Monotherapy\# & -49.83 & -3.35 & $<0.01$ \\
\hline Combination therapy & -37.66 & -2.34 & 0.03 \\
\hline \multicolumn{4}{|c|}{$\begin{array}{l}\text { Gender0: female, Gender1: male gender, * : the basic group is SLE } \\
\text { group, \#: the basic group is untreated group; CTD: connective tissue } \\
\text { disease, PAH: pulmonary arterial hypertension. SLE: systemic lupus } \\
\text { erythematosus, MCTD: mixed connective tissue disease, SS: } \\
\text { Sjogren's syndrome, SSc: systemic sclerosis, BMI: body mass index }\end{array}$} \\
\hline
\end{tabular}

shows the rise of PAP, then the patients should do a RHC examination to confirm the diagnosis.

Immunosuppressive treatment is effective for improving symptoms and hemodynamic parameters in some patients with CTDPAH, especially in those with active-phase SLE or MCTD [14-16]. Despite these short-term effects, immunosuppressive treatment did not improve long-term survival. Therefore, the effects of immuno- suppressive treatment are likely limited, and pulmonary vasodilation therapy should be used in combination with immunosuppressive treatment for CTD-PAH. As shown in the results, both univariate and multivariate analyses revealed that pulmonary vasodilation therapy could significantly improve mPAP.

There is evolving evidence to suggest that, compared with monotherapy, the treatment of PAH with combination therapy is associated with improved survival in patients with PAH. In some small randomized trials and observational studies, combination therapy by means of 'add-on' PAH therapy has consistently shown a survival benefit in patients with PAH [17-19]. Recently, the treatment of PAH with combination therapy compared with monotherapy showed not only a survival benefit but also reduced hospitalization for worsening PAH and disease progression [20]. However, in our study, we did not notice that the combination therapy showed better improvement of mPAP compared with monotherapy. We believe that limited data might account for this. In addition, patients who received combination therapy had a higher mPAP at PAH diagnosis than those who received monotherapy.

Some limitations should also be considered in this study. First, it is ideal to collect clinical data at the time of disease diagnosis to better correlate disease pathology with risk factors. However, it is challenging to obtain these concurrent data in practice, especially in China. In our study, a subset of clinical data was obtained at disease diagnosis, but most of the data were obtained at the relapse or remission stage. In addition, as mentioned before, most patients will not go to the hospital until right ventricular dysfunction has developed. In conclusion, the underlying CTDs in our patients with CTD-PAH were completely different from those in the USA and Europe. Modern pulmonary vasodilation treatment improves survival rates. Early detection of PAH in Chinese patients with CTD is important for further improving survival rates.

\section{Competing interest}

The authors declare that they have no conflict of interest.

\section{References}

[1] Trad S, Amoura Z, Beigelman C et al. Pulmonary arterial hypertension is a major mortality factor in diffuse systemic sclerosis, independent of interstitial lung disease. Arthritis and rheumatism 2006; 54: 184-191

[2] Chung L, Liu J, Parsons L et al. Characterization of connective tissue disease-associated pulmonary arterial hypertension from REVEAL: identifying systemic sclerosis as a unique phenotype. Chest 2010; 138 : 1383-1394

[3] Clements P], Tan M, McLaughlin VV et al. The pulmonary arterial hypertension quality enhancement research initiative: comparison of patients with idiopathic PAH to patients with systemic sclerosis-associated PAH. Annals of the rheumatic diseases 2012; 71: 249-252

[4] Condliffe R, Kiely DG, Peacock AJ et al. Connective tissue disease-associated pulmonary arterial hypertension in the modern treatment era. American journal of respiratory and critical care medicine 2009; 179: $151-157$ 
[5] Ruiz-Cano M], Escribano P, Alonso R et al. Comparison of baseline characteristics and survival between patients with idiopathic and connective tissue disease-related pulmonary arterial hypertension. The Journal of heart and lung transplantation : the official publication of the International Society for Heart Transplantation 2009; 28: 621-627

[6] Launay D, Humbert M, Berezne A et al. Clinical characteristics and survival in systemic sclerosis-related pulmonary hypertension associated with interstitial lung disease. Chest 2011; 140: 1016-1024

[7] Denton CP, Humbert M, Rubin L et al. Bosentan treatment for pulmonary arterial hypertension related to connective tissue disease: a subgroup analysis of the pivotal clinical trials and their open-label extensions. Annals of the rheumatic diseases 2006; 65: $1336-1340$

[8] Quan H, Qi L, Luo X et al. Considerations of multiple imputation approaches for handling missing data in clinical trials. Contemporary clinical trials 2018; 70: 62-71

[9] Chung SM, Lee CK, Lee EY et al. Clinical aspects of pulmonary hypertension in patients with systemic lupus erythematosus and in patients with idiopathic pulmonary arterial hypertension. Clinical rheumatology 2006; 25: 866-872

[10] Shirai Y, Yasuoka H, Okano Y et al. Clinical characteristics and survival of Japanese patients with connective tissue disease and pulmonary arterial hypertension: a single-centre cohort. Rheumatology (Oxford, England) 2012; 51: 1846-1854

[11] Zhang R, Dai LZ, Xie WP et al. Survival of Chinese patients with pulmonary arterial hypertension in the modern treatment era. Chest 2011; 140: 301-309

[12] Kim WU, Min JK, Lee SH et al. Causes of death in Korean patients with systemic lupus erythematosus: a single center retrospective study. Clinical and experimental rheumatology 1999; 17: 539-545
[13] Lau EM, Manes A, Celermajer DS et al. Early detection of pulmonary vascular disease in pulmonary arterial hypertension: time to move forward. European heart journal 2011; 32: 2489-2498

[14] Miyamichi-Yamamoto S, Fukumoto $Y$, Sugimura K et al. Intensive immunosuppressive therapy improves pulmonary hemodynamics and long-term prognosis in patients with pulmonary arterial hypertension associated with connective tissue disease. Circulation journal: official journal of the Japanese Circulation. Society 2011; 75: 2668-2674

[15] Sanchez O, Sitbon O, Jais X et al. Immunosuppressive therapy in connective tissue diseases-associated pulmonary arterial hypertension. Chest 2006; 130: 182-189

[16] Kato M, Kataoka H, Odani T et al. The short-term role of corticosteroid therapy for pulmonary arterial hypertension associated with connective tissue diseases: report of five cases and a literature review. Lupus 2011; 20: 1047-1056

[17] Ngian G, Nikpour M, Byron J et al. Survival in Australian patients with connective tissue disease-associated pulmonary arterial hypertension. Internal medicine journal 2011; 41: 12

[18] Simonneau G, Rubin L], Galie N et al. Addition of sildenafil to long-term intravenous epoprostenol therapy in patients with pulmonary arterial hypertension: a randomized trial. Annals of internal medicine 2008; 149: 521-530

[19] Ghofrani HA, Galie N, Grimminger F et al. Riociguat for the treatment of pulmonary arterial hypertension. The New England journal of medicine 2013; 369: 330-340

[20] Galie N, Barbera JA, Frost AE et al. Initial Use of Ambrisentan plus Tadalafil in Pulmonary Arterial Hypertension. The New England journal of medicine 2015; 373: 834-844 